\title{
Hematogones in the bone marrow of a child with Rubella virus-associated immune thrombocytopenic purpura concomitant with iron deficiency anemia
}

\author{
Demir eksikliği anemisine eşlik eden Rubella virüsüne bağlı immune \\ trombositopenik purpuralı bir çocuğun kemik iliğindeki hematogonlar
}

Faruk Barlık ${ }^{1}$, Emel Özyürek², Feride Duru²

${ }^{1}$ Department of Pediatrics, Faculty of Medicine, Ondokuz Mayıs University, Samsun, Turkey

2Department of Pediatrics, Hematology Section, Faculty of Medicine, Ondokuz Mayı University, Samsun, Turkey

Hematogones are normal B-cell precursors that are observed in small numbers in the bone marrow; however, significant expansion of hematogones can occur in the bone marrow in healthy newborns, and in individuals with malignant and non-malignant hematologic disorders, including congenital and autoimmune cytopenias, nutritional anemias, viral infections, and Gaucher disease [1-6]. Morphologically, hematogones are small- to medium-sized cells with condensed chromatin and scant cytoplasm devoid of inclusions and vacuoles; some hematogones resemble leukemic lymphoblasts. As the morphological and immunophenotypical features of hematogones are similar to those of the blasts in precursor B acute lymphoblastic leukemia (ALL), sometimes diagnostic problems arise. Using flow cytometry hematogones can be identified by their peculiar antigenic profile; 3 forms are defined on the basis of their maturational stage [1-6].

Stage 1 hematogones are the most immature form, and along with CD34 and TdT positivity they exhibit dimmer expression of CD45 and CD19, and brighter expression of CD10, as compared to the more mature forms and normal lymphocytes. Stage 1 hematogones lack CD20. Stage 2 hematogones represent the majority of the hematogone popula- tion. They exhibit high CD34 and TdT experession, with increased expression of CD19, dim expression of CD20, and gradually decreasing expression of CD10. As they mature to stage 3 they exhibit brighter expression of CD20 and CD45, and dimmer expression of CD10, as compared to the more immature forms of hematogones. Recently, stage 3 and some stage 2 hematogones were reported to express CD5 concomitantly with CD19 and CD10. Dim expression of CD22 and bright expression of CD38 are seen in each hematogone stage [7].

A 1-year-old girl presented with a cough and a rash on her body $1 \mathrm{~d}$ after developing fever. Her medical and family histories were non-contributory. On physical examination she had petechiae all over her body and on both tonsils. She did not have organomegaly. The remaining systemic examinations were normal. Her complete blood count results were as follows: hemoglobin: $9.3 \mathrm{~g} \mathrm{dL}^{-1}$; hematocrit: $27 \%$; erythrocyte count: $4.01 \times 10^{9} \mathrm{~L}^{-1}$; mean corpuscular volume: $69 \mathrm{fL}$; red cell distribution width: 15.5\%; white blood cell count: $14,100 \mathrm{~mm}^{-3}$; platelet count: $2000 \mathrm{~mm}^{-3}$. There were $80 \%$ lymphocytes, $20 \%$ neutrophils, 1 or 2 platelets without aggregation, and hypochromic microcytic erythrocytes on 
peripheral blood smear. We observed 12\% mononuclear cells resembling lymphoblasts (Figure 1). Immunophenotypic analysis of her bone marrow was performed using flow cytometry. Total lymphoid cells were selected using SSC versus FSC gating (Figure 2a). B-lymphoid cells (48\%-49\% of total lymphocytes) expressing CD19 and CD22 were observed. Among all the lymphocytes (Figure 2a), those constituting 38\% that showed bright CD10 expression, and coexpression of CD19 and CD5 (Figure 2b and c) were considered as hematogones.

A small number (approximately 4\%) of cells that were probably early-stage or stage 1 hematogones also expressed CD34 and TdT (Figure 2d and e). These cells lacked aberrant marker expression. Serology was positive for rubella virus immunoglobulin M. Iron panel of the patient showed iron deficiency anemia (serum iron level: $20.6 \mu \mathrm{g} \mathrm{dL}^{-1}$; serum iron binding capacity: $460 \mu \mathrm{g} \mathrm{dL}^{-1}$; transferrin saturation: 5.8\%; ferritin: $5.8 \mathrm{ng} \mathrm{dL}^{-1}$ ) and complete blood count parameters in both of the patient's parents were normal. As such, rubella virus-associated immune thrombocytopenic purpura was considered as the patient's diagnosis, in addition to iron deficiency anemia.

The patient was given intravenous immunoglobulin $\left(2 \mathrm{~g} \mathrm{~kg}^{-1}\right)$ and ferrous sulphate treatments. An significant increase in her platelet count was observed within 2 days. A follow up bone marrow smear performed 2 days after the first one or immediately following the therapy showed a decrease in the number of hematogones (3\%) by morphological examination as compared to the previous analysis. But flow cytometric analysis of the second bone marrow sample was not performed/done at that time. She was discharged with a platelet count of

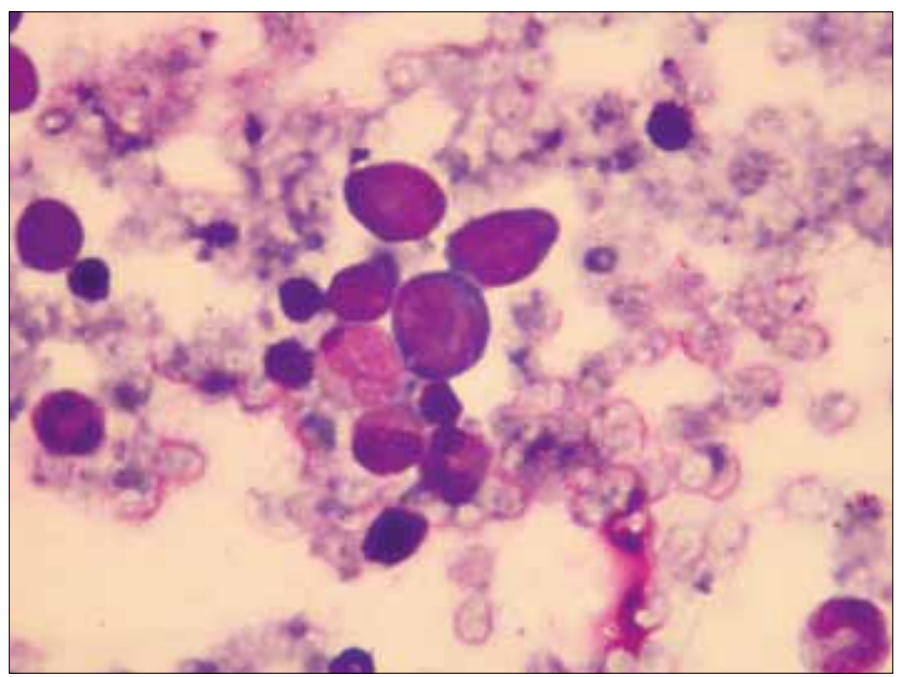

Figure 1. Hematogones with blast-like features in the bone marrow sample
$153,000 \mathrm{~mm}^{-3}$. One month later her hemoglobin increased to $12 \mathrm{~g} \mathrm{dL}^{-1}$, and the ferrous sulphate dose was decreased accordingly. She received iron therapy for a total of 3 months starting from her hospitalisation. During 6 months of follow-up her thrombocyte count fluctuated between 112,000 and $451,000 \mathrm{~mm}^{-3}$.

The presented patient had rubella virus-associated thrombocytopenia and iron deficiency anemia; both conditions are reported to be associated with the appearance of hematogones [7]. Once the diag-

a

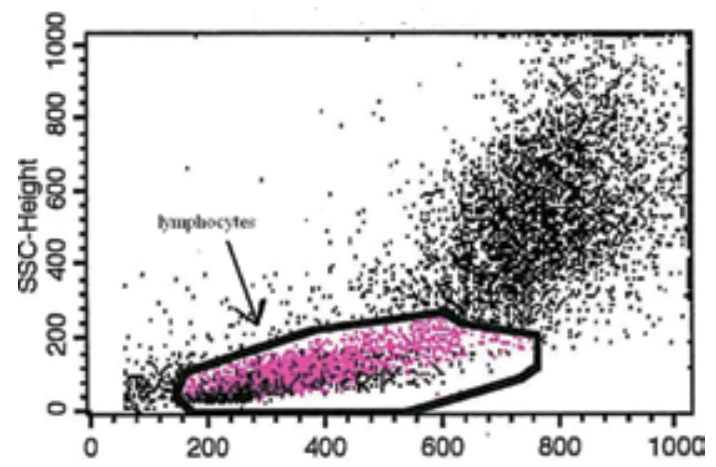

b

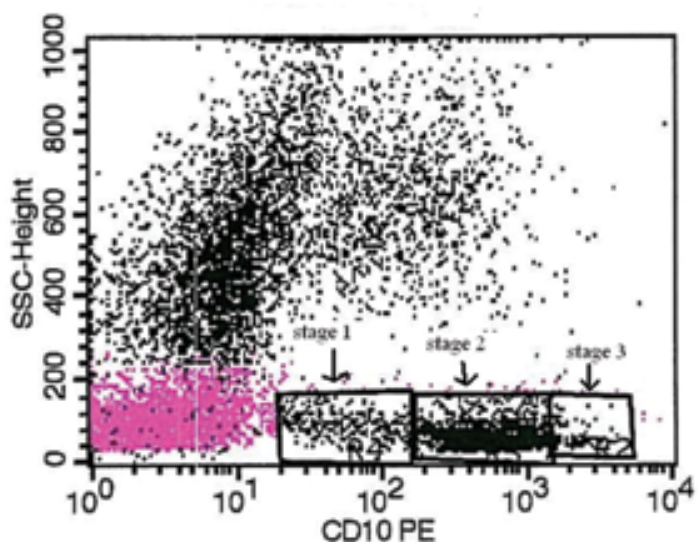

C

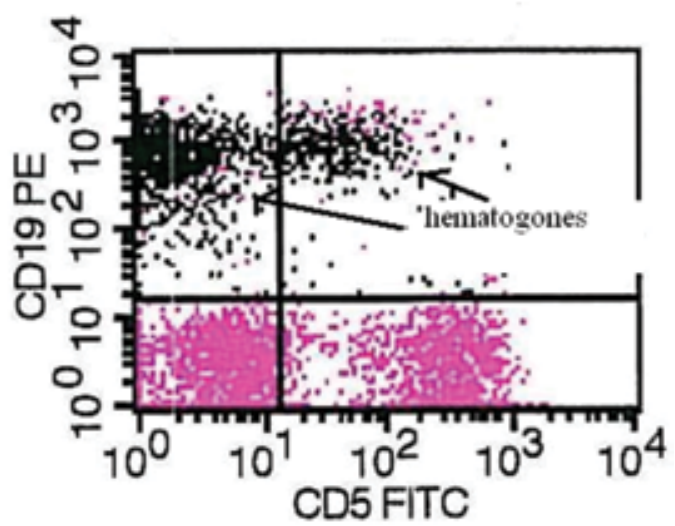



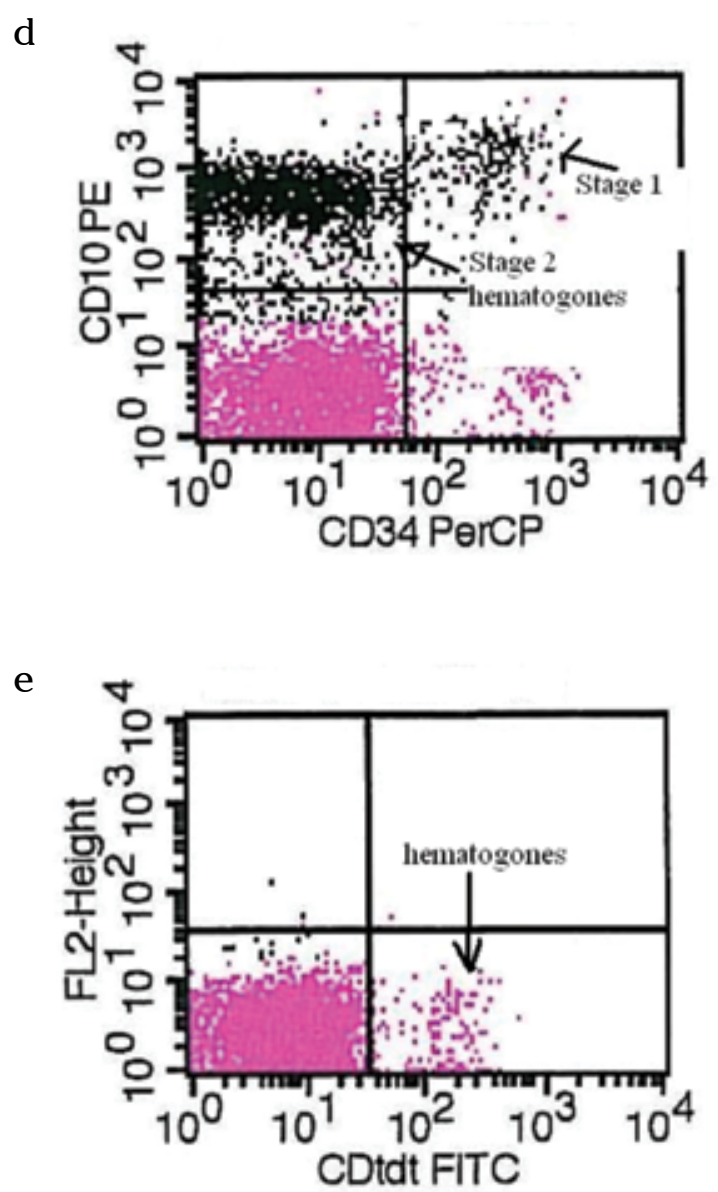

Figure 2. a. On the SSC-FSC scatter plot we gated the total lymphocyte population. b. In the SSC versus CD10 plot there were 3 different populations of hematogones (in stage 1, stage 2 , and stage 3 , shown with arrows) with very low side scatter and variable expression of CD10. c. Hematogones with bright expression of CD19 and CD5. d. Cells shown with arrows are early-stage or stage 1 hematogones with CD10 and CD34, as well as stage 2 hematogones expressing CD10, but lacking CD34. e. Early hematogones expressing TdT (arrow)

nosis was made and appropriate therapy was initiated, rapid improvement of thrombocytopenia along with a striking reduction in bone marrow hematogones was observed. The cause of the observed rapid reduction in hematogones is not clear, but may have been due to their differentiation into more mature forms-probably due to the effect of high-dose intravenous immunoglobulin and/or iron therapy.

Hematogones are B-cell precursors that exhibit a continuous and complete maturation spectrum. In the presented case stage 2 hematogones comprised the majority of the hematogone population, as was reported in most other cases [7]. The most mature stage 3 hematogones resemble mature lymphocytes and frequently remain unrecognized, whereas less mature forms-predominantly stage 1 and some stage 2 hematogones-resemble blasts of ALL. In the morphological examination of the second bone marrow, we observed a significant decrease in the percentage of blast-like cells which were identified as stage 1 and some stage 2 hematogones (12\%) by immunophenotypic analysis of the first marrow specimen. This prompt fall in the number of blast-like cells may be explained by the maturation of those early stage hematogones to stage 3 ones which are difficult to differentiate by histopathological examination of a marrow smear.

In conclusion, excessive hematogones in the bone marrow of children may cause problems in diagnostically differentiating acute lymphoblastic leukemia from a benign hematogone proliferative state, and flow cytometric analysis may help differentiate these 2 cell populations.

Written informed consent was obtained from the patient.

\section{Conflict of interest statement}

The authors of this paper have no conflicts of interest, including specific financial interests, relationships, and/or affiliations relevant to the subject matter or materials included.

\section{References}

1. McKenna RW, Washington LT, Aquino DB, Picker LJ, Kroft SH. Immunophenotypic analysis of hematogones (B-lymphocyte precursors) in 662 consecutive bone marrow specimens by 4-color flow cytometry. Blood 2001;98:2498-507.

2. Rimsza LM, Douglas VK, Tighe P, Saxonhouse MA, Calhoun DA, Christensen RD, Sola MC. Benign B-cell precursors (hematogones) are the predominant lymphoid population in the bone marrow of preterm infants. Biol Neonate 2004;86:247-53.

3. Babusíková O, Zelezníková T, Kirschnerová G, Kankuri E. Hematogones in leukaemia during and after therapy. Leuk Lymphoma 2008;49:1935-44.

4. van Lochem EG, Wiegers YM, van dem Beemd R, Hahlen K, van Dongen JJM, Hooijkaas H. Regeneration pattern of precursor-B cells in bone marrow of acute lymphoblastic leukaemia patients depends on the type of preceding chemotherapy. Leukemia 2000;14:688-95.

5. Vargas SO, Hasegawa SL, Dorfman DM. Hematogones as an internal control in flow cytometric analysis of suspected acute lymphoblastic leukaemia. Pediatr Dev Pathol 1999;2:371-6.

6. Fisgin T, Yarali N, Duru F, Kara A. CMV-induced immune thrombocytopenia and excessive hematogones mimicking an acute B-precursor lymphoblastic leukaemia. Leuk Res 2003;27:193-6.

7. Sevilla DW, Colovai AI, Emmons FN, Bhagat G, Alobeid B. Hematogones: a review and update. Leuk Lymphoma 2010;51:10-9. 